\title{
Online Misinformation Analysis through Wittgensteinian Lens
}

\author{
Uyiosa Omoregie
}

August 2021

Avram Turing, Guelph, ON, Canada

uyiosa.omoregie@avramturing.com

The works of Ludwig Wittgenstein (1889-1951) are usually divided into two: the early and the later philosophy. Wittgenstein's book Tractatus Logico-Philosophicus [1] is regarded as his early masterpiece. Another book Philosophical Investigations [2] is representative of his later philosophical work. Tractatus Logico-Philosophicus (TL-P) is concerned with the role facts play in the world. According to TL-P, the world is composed of facts and we connect with facts by our thoughts. Our thoughts picture the world and are expressed through propositions. In Philosophical Investigations (PI) Wittgenstein refutes most of what he stated in TL-P and goes on to criticise all traditional philosophy. In PI Wittgenstein suggests that the misuse of language or conceptual confusions are at the heart of the problems of philosophy.

In TL-P, Wittgenstein expresses a belief in 'analysis': the breaking down of thought or propositions into basic components for better understanding (which Russell [3] termed 'logical atomism'). But, according to Russell [3], by the time he wrote PI Wittgenstein no longer believed in analysis: he changed his approach from analysis to criticism. TL-P remains an important book and is highly regarded today. A poll of 414 philosophy teachers, in North America, revealed TL-P to be among the top five books of philosophy written in the twentieth century [4].

We found no previous work that use Wittgenstein's early philosophy for misinformation analysis. Giulietti [5] used Wittgenstein's later philosophy of language (presented in PI) to reveal how a popular politician successfully changed "the language game of politics" with devastating effects on truth and democracy. In Giulietti's analysis this politician played the language game using irony, sarcasm and outright lies successfully. This was possible because, according to Wittgenstein's Pl, the meaning of language depends strictly on context. One man's "fact" could be another man's lie if the game is played skilfully. For example, the expression "I do not hate my political enemies, I love them too much" could mean one thing for the speaker's allies and mean something else to his competitors. For misinformation analysis however, the PI would not be adequate as a framework, we must instead turn to TL-P for insights. In TL-P, Wittgenstein states that philosophy should aim for nothing more than "the logical clarification of thoughts."

TL-P is composed of seven main theses and accompanying remarks (or sub-theses) on these theses. Within the seven main theses, Wittgenstein tries to get at the heart of the problems of philosophy by explaining how language really works. He does not succeed in this ambitious quest. His theory in TL-P cannot apply to language as a whole (unlike the theory in $\mathrm{Pl}$ ). But, in reaching to solve the highest issues, Wittgenstein provides insights into the nature of the world of communication and information. In TL-P we find a useful theory about declarative sentences and propositions. Some principal theses (relevant to this paper) in TL-P can be summarised thus:

I. Facts are the real world

The thesis number " 1 " is the first of seven major theses (1-7). Three of its propositions:

1. The world is everything that is the case.

1.11 The world is determined by the facts, and by these being all the facts. 


\subsection{The world divides into facts.}

Wittgenstein states that facts divide the world: they determine everything that exists or does not exist in the world. Facts are derived from 'states of affairs'. States of affairs that exist prove those states that do not exist.

\section{II. $\quad$ Language consists of propositions which could represent the world}

2.19 The logical picture can depict the world.

3. The logical picture of the fact is the thought.

4. The thought is the significant proposition.

4.001 The totality of propositions is the language.

Wittgenstein declares that the world can be pictured or represented by propositions. Every proposition is either true or false. All language is made up of propositions and it can only represent the world correctly when its propositions are true. A logical picture of a fact is a thought and thoughts find expression through propositions. Every proposition has only one complete analysis and a fact has only one correct analysis. Only factual discourse contains reasoning.

\section{Logic fills the world and limits it}

Wittgenstein describes the importance of logic in some sub-theses/remarks:

5.61 Logic fills the world: the limits of the world are also its limits.

6.124 The logical propositions describe the scaffolding of the world, or rather they present it.

6.13 Logic is not a theory but a reflection of the world.

Logic aims to present clearly what can be inferred from a proposition. What cannot be inferred from a proposition is regarded as not valid.

IV. Some things are better left unsaid and cannot be analysed rationally

Wittgenstein's last thesis consists of just one line with no sub-theses or remarks:

7. Whereof one cannot speak, thereof one must be silent.

Wittgenstein implies here that there are certain things/phenomena which cannot be properly expressed through language. These things can be described as mystical/mysterious or simply unsayable. They can be known or shown but not properly articulated. The rules described by Wittgenstein in the preceding six major theses cannot apply to such unspeakable things/phenomena.

In summary, there are four issues in Wittgenstein's Tractatus Logico-Philosophicus that are of relevance to online misinformation analysis: 'Facts' 'Propositions' 'Logic/Analytical' and the 'Unspeakable'.

The system for online content analysis, described here, is a descriptive tool (see figure below). This tool aims to clarify the thoughts and propositions found within the content analysed. In this Wittgensteinian model, Web-based written non-graphical information (articles, commentary etc.) is analysed and then scored based on criteria designed to evaluate the quality of the content. Post-analysis, the written content is then categorised as 'analytical' or 'non-analytical'. Further labelling of the intrinsic nature of the content (e.g. 'satire' 'political' 'scientific') and users' (content consumers) ratings completes the process. Our hypothesis is that when applied to Web browsers and online social media platforms, the rating produced by the information quality analysis system will help users discern content qualitatively and engage more analytically with other users. 
At the heart of this online information quality model is the belief that analytical thinking, (clarification of thoughts and propositions), is essential for combating information disorder and the lure of echo chambers [6]. This model applies the four aspects of the Wittgensteinian framework (mentioned in section 2 above) consisting of: (a) facts (b) propositions (c) logical/analytical thinking (d) labelling unanalysable content.

Online written content is first fact-checked. The second step is to screen content for propositions. The third step scores the content as 'analytical' or 'non-analytical': the score depends on the coherence and logical validity of the content's propositions. The final step is labelling of the content as a whole. This step is important because conspiracy theories and other such beliefs are not analysed but labelled. Such beliefs are regarded as mysterious, they can only be made manifest or shown and are better left unspoken, in line with the Wittgensteinian philosophy (LT-P thesis 7.).

Web browser extensions can be offered to users that will reveal automatically the analytical rating of a trending article, tweet or other written content. This rating is activated once a link to the content is accessed. Search engines will display search results that include a score for any trending written content found in the search results. Users of social media platforms like Facebook, Twitter etc. will see the analytical quality score of trending content posted on their newsfeeds. These features may encourage the promotion of quality content online. Hopefully, as more users recognise quality content and discern its features, they should increasingly re-post, re-tweet and forward such content, leading in turn to increased user engagement with quality content.

Sharot [7] suggests that more incentives from social media platforms are required for online content consumers to engage more with 'truth' online. More 'carrots' are needed to compliment 'sticks'. A visible high rating (explained above) displayed for quality content can act as a carrot rewarding the creation of more quality content and increased engagement with such content. As Donovan emphasizes, it is important that 'authoritative content' is elevated online [8]. The approach to misinformation prevention and analysis explained in this paper does this by targeting the virality of content, which is a priority for social media platforms [9]. Research shows that interventions that help shift people's attention towards a measure of the accuracy (quality) of content they encounter online encourages them to share higher quality news, thereby reducing the chance of others being misled my misinformation [10]. Another study reveals that social media algorithms could reduce the spread of misinformation by elevating ('up-ranking') content from trusted media websites, and 'down-ranking' content from websites with low trustworthiness (low information quality) ratings [11].

Further research will be required to confirm or refute the hypotheses we have stated and answer the implied research questions from this paper. 


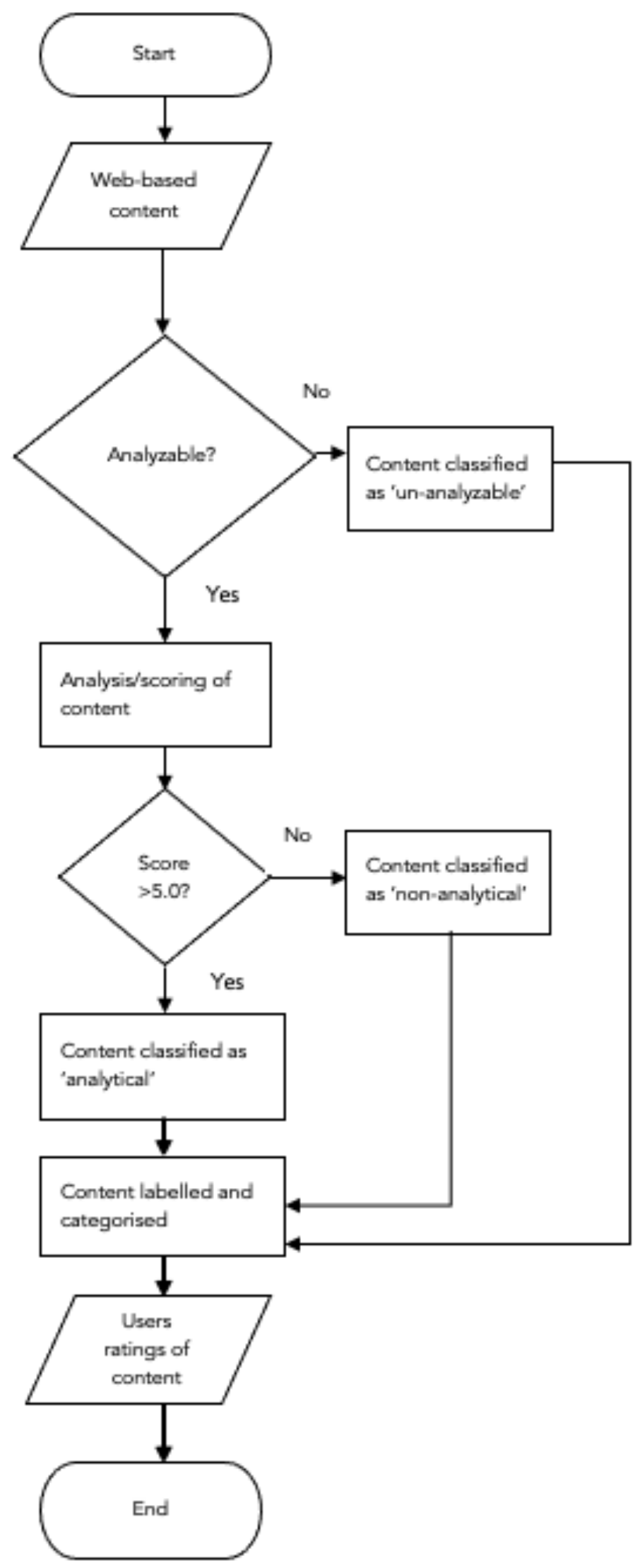

Figure: Flowchart for Wittgensteinian analysis of the information quality of online content 


\section{References}

[1] Wittgenstein, L. Tractatus Logico-Philosophicus. London: Paul, Trench, Trubner \& Co., 1922.

[2] Wittgenstein, L. Philosophical Investigations. Chichester: Wiley-Blackwell, 1953.

[3] Russell, B. My Philosophical Development. Bombay: Affiliated East-West Press, 1959, p.118.

[4] Lackey, D. What are the modern classics? The Baruch poll of great philosophy in the twentieth century. The Philosophical Forum 1999; 30: 329-346.

[5] Giulietti, P. The hidden danger of trump: how Trump changed the language game of politics and its effect on truth and democracy. Liberated Arts; 4(1),

https://ojs.lib.uwo.ca/index.php/lajur/article/view/7266

[6] Swami, V, Voracek, M, Steiger, S, et al. 2014. Analytic thinking reduces belief in conspiracy theories. Cognition 2014; 133: 572 - 585.

https://www.sciencedirect.com/science/article/abs/pii/S0010027714001632

[7] Sharot, T. To quell misinformation, use carrots-not just sticks. Nature. 2021; $591(7850): 347$

[8] Donovan, J. Social-media companies must flatten the curve of misinformation. Nature, https://www.nature.com/articles/d41586-020-01107-z

[9] Mims, C. Online speech is now an existential question for tech, The Wall Street Journal. https://www.wsj.com/articles/online-speech-is-now-an-existential-questionfor-tech-11613797254

[10] Pennycook, G, Epstein Z, Mosleh M, Arechar A, Eckles D and Rand D. Shifting attention to accuracy can reduce misinformation online. Nature 2021; 17:1-6.

[11] Pennycook, G, Rand, D. Fighting misinformation on social media using crowdsourced judgments of news source quality. P Natl Acad Sci. 2019; 116(7):2521-6. 